\title{
Report on the Sixth Conference on Artificial General Intelligence
}

\author{
Kai-Uwe Kühnberger, Sebastian Rudolph, Pei Wang
}

The Sixth Conference on Artificial General Intelligence (AGI-13) was held from July 31 to August 3, 2013, in Beijing, China. This report summarizes the major events during the conference, as well as the main topics addressed.

\begin{abstract}
$\Lambda$ rtificial general intelligence (AGI) is concerned with the attempt to build systems that show intelligence on human level and scale while working in realistic situations, that is, systems that can operate in environments they do not know in advance, on tasks they are not specifically designed for, and that embrace the breadth of human capabilities. Motivated by the original idea of artificial intelligence in the 1950s and 1960s, there has been a revival of research in general intelligence during the last years. The annual AGI conference series, which is the major event in this area, has been held in cooperation with AAAI since 2008.

The sixth conference on AGI was held at Peking University, Beijing, from July 31 to August 3, 2013. AGI-13 was collocated with the International Joint Conference on Artificial Intelligence (IJCAI 2013), the major international AI conference. This was the first time an AGI conference took place in Asia. A major reason for this was to promote AGI research in the emerging and upcoming eastern countries. All in all, it
\end{abstract}


can be said that it was a great success. The authors of the submitted papers came from 23 different countries and the participants of the conference were equally international. The facilities of Peking University provided a nice environment that promoted lively discussions. On the conference website ${ }^{1}$ interested readers can find the videos and slides of the presentations and discussions, as well as all the papers collected in the conference proceedings.

\section{Main Conference}

Following the established tradition of AGI conferences, all presentations were held in a single track. Although unusual for an AI conference, the single-track presentation has turned out to be rather appropriate for the AGI community, because it supports and facilitates discussions, prompts inspirations from different fields, and allows participants to learn more about research methodologies that are not in the focus of one's own history.

The presentations of the regular papers were clustered into four technical sessions: AGI architectures and cognitive systems, learning in AGI systems, programming and natural language systems, and theoretical and conceptual issues of AGI systems.

\section{AGI Architectures and Cognitive Systems}

This cluster can be called a classical field of AGI research. Focusing on general architectures sheds light on the implementation and instantiation of a broad variety of cognitive abilities, the integration aspect of different methodologies, as well as certain technical results on some of the frameworks used for AGI applications.

\section{Learning in AGI Systems}

Rather similar to classical AI conferences in which learning abilities of systems and agents are considered to be important, the session on learning emphasized the crucial learning aspect of models for general intelligence. Nevertheless, the presented methods were nonstandard in the sense that no detailed results about popular learning methods (like Bayesian learning or kernel methods) were presented, but fresh approaches for learning were proposed. For example, learning by combining combinatorial logic with genetic programming, learning from experience and problem solving, or the integration of probabilistic logic, frequent pattern mining, and deep learning were presented.

\section{Programming and}

\section{Natural Language Systems}

The session about languages covered the two word senses of "language" in AI research - first, the scientific study of programming languages, and second, the modeling and analysis of natural language. Although this might appear to be a balancing act, because all in all the two topics are rather different concerning their domain and the used methods, this session turned out to be quite inspiring. The participants heard interesting talks about, for example, innovative approaches to natural language processing, a new programming paradigm of control systems for robotic applications (Replicode), and Lojban++, a language that has the potential to bridge the gap between programming languages and natural languages.

\section{Theoretical and Conceptual Issues of AGI Systems}

This session spanned from tractability issues of AI and the role of (cognitively and psychologically inspired) constructions in general intelligence to embodiment issues of AGI. Furthermore, the alignment of stochastic and continuous real-world environments on the one hand and discrete state spaces and deterministic modelings (as often required in theories for decision making) on the other, were examined. In this session, not only technical results were presented but also several conceptual issues were raised.

\section{Special Sessions}

In addition to the four technical sessions sketched above, two special sessions were organized, both of them with a strong relation to and a potentially high impact on the Asian world: Cognitive Robotics and AGI and AGI in Asia. Several novel approaches toward AGI were introduced and discussed.

\section{Prizes}

At the end of AGI-13 three prizes were awarded. Two Kurzweil prizes (sponsored by KurzweilAI.Net), one for the best paper of the conference and one for the best AGI-related idea, have already a certain tradition in the AGI series, as they have been awarded in the last AGI conferences as well. The third prize was newly sponsored by the Cognitive Science Society. This prize was awarded to the best student paper.

Computational Versions of Kolmogorov Complexity Based on Cognitive Models, by C. Strannegard, A. Nizamani, A. Sjöberg, F. Engström, was selected as the winner of the Kurzweil Best AGI Paper. Resource-Bounded Machines Are Motivated to Be Effective, Efficient, and Curious, by B. Steunebrink, J. Koutník, K. Thórisson, E. Nivel, and J. Schmidhuber, was selected as the winnter of the Kurzweil Best AGI Idea Paper. Where Almost Is Not Even Close: Remarks on the Approximability of HDTP, by T. Besold and R. Robere, was selected as the winner of the Best AGI Student Paper.

\section{Keynotes}

As is commonplace for international conferences, important highlights were the keynote talks. At AGI-13, Stuart Shapiro (professor emeritus of the University of Buffalo), Thomas G. Dietterich (University of Oregon), and Dileep George (Vicarious Systems, Inc.) gave inspiring talks about their view of artificial general intelligence.

Stuart Shapiro presented in his keynote a Modal Grounded Layered Architecture with Integrated Reasoning, which can be considered as a typical AGI project, given the cognitive functions involved. This architecture was exemplified using various agents ranging from Wumpus World Agents to cleaning robots and delivery agents. Furthermore, Shapiro presented details of the architecture itself: for example, he explained the perceptuo-motor layer, the knowledge representation system SNePS 2.8, and the supported reasoning mechanisms. In total, the talk showed a general framework that can be instantiated for various application domains in order to show general forms of intelligence. 
Thomas G. Dietterich, presidentelect of AAAI, presented details about CALO (cognitive assistant that learns and organizes), a multi-institutional cooperation on an electronic secretary. The important aspect in this project that is targeted at general intelligence is the integration of many abilities: CALO is able to support the user in tasks like email management, scheduling, summarizing meetings, project management, presentations, and many more tasks that appear quite regularly in administrative types of work of many institutions. In order to enable the system to do that, CALO integrates many classical challenges of AI research like rudimentary natural language processing abilities, learning mechanisms (such as for profiling users), new interface design types for intuitive usability, appropriate knowledge representation formalisms, handling of uncertainty, and many more. Beside the design of CALO, the talk also summarized the lessons learned from its building.

Dileep George suggested building AGI "the brain way." He explained the neuroscientific foundation of several cognitive functions, as well as the way to model them in computer systems. He suggested that the mechanism found in neocortex is both general and efficient, and it is based on hierarchical structure. It is argued that a brainlike system should be built starting from perception and action rather than from high-level cognitive function like reasoning or planning.

\section{Workshops and Tutorials}

Following the tradition of AGI conferences the framing days of the main conference were dedicated to workshops (July 31) and tutorials (August $3)$. Another tradition of AGI conferences is that workshops and tutorials are not held in parallel sessions but, in a manner similar to the main conference, are organized in a single-track structure. This ensures a large number of participants in the workshops and tutorials and is a pleasant contrast to sometimes rather sparsely populated workshops at large AI conferences.

The two workshops that were offered at AGI-13 were Formal Magic:
Formalizing Mechanisms for Artificial General Intelligence and Cognition, and Probability Theory or Not? Practical and Theoretical Concerns on Uncertainty Handling in AGI. Both workshops addressed important challenges of AGI research, namely the formally sound foundation of any AGI-relevant approach and an assessment of the currently popular probabilistic direction of AI and AGI research. Both challenges are important: if it is true that it is time to develop a new research paradigm for AGI, then a thorough formal basis is necessary for progress. Concerning the second workshop, it is important to raise the question to which extent the current popularity of probability-based methods in AI research could also be a reasonable choice for AGI research. The papers presented at the two workshops contributed important aspects to the main discussions in the sketched fields of challenges. Additionally, the organizers augmented the workshops with invited lectures that triggered fruitful discussions that may affect future AGI research.

The tutorials were taught by Stuart Shapiro and Marcus Hutter. Shapiro explained his MGLAIR architecture more thoroughly, and Hutter reported his perspective on a decade of universal artificial intelligence with the AIXI model and its extensions. The tutorials highlighted directions of AGI research, namely the reinforcement learning approach enriched with insights from Kolmogorov complexity, data compression, and information theory on the one side and the extensive work on cognitive architectures and cognitive systems on the other.

\section{Conclusion}

The Sixth Conference of Artificial General Intelligence was a well-organized, successful conference that took place in the center of the emerging Asian scientific hightech culture. The conference included many inspiring presentations that, we hope, will encourage future researchers in Asia to participate in and contribute to this line of research. The emerging Asian research community can make a difference in developing AGI in the future.

\author{
Note \\ 1. See www.agi-conference.org/2013.
}

Kai-Uwe Kühnberger is a university professor and dean of the faculty of human sciences at the Institute of Cognitive Science of the University of Osnabrück. He received his Ph.D. in computational linguistics from the University of Tübingen in 2002. His main research areas are nonclassical reasoning, ontologies, and text technology, computational creativity, and cognitively inspired learning mechanisms. $\mathrm{He}$ is editor-in-chief of the Springer series Thinking Machines and survey editor of Cognitive Systems Research. He was a program committee cochair of AGI-13.

Sebastian Rudolph is university professor for computational logic at the AI Institute of the Technische Universität Dresden. He received his Ph.D. in mathematics from that university in 2006. His main research focuses are the modeling and reasoning aspects of logic-based knowledge representation, ranging from the mathematical and formal foundations of diverse knowledge representation paradigms to their applications to areas like the semantic web. He serves on the editorial boards of the Journal of Web Semantics and the Journal on Data Semantics and on the steering committees of several AI-related conferences. He was a program committee cochair of AGI-13.

Pei Wang is an associate professor at Temple University. He received his B.S. degree and M.S. degree in computer science from Peking University, in 1983 and 1986, respectively, and his Ph.D. degree in computer science and cognitive science from Indiana University, Bloomington, in 1995. His research focuses on unified theories of intelligence, formal models of rationality, reasoning under uncertainty, learning and adaptation, and real-time decision making. He was the conference chair of AGI-13. 